\title{
Controlled release study of an anti-carcinogenic agent, gallate from the surface of magnetite nanoparticles.
}

\begin{abstract}
Immobilization of gallate anion, an anti-carcinogenic, anti-mutagenic, and anti-microbial agent on the surface of magnetite nanoparticles was accomplished by adsorption technique for the formation of a core-shell nanocomposite. A simple co-precipitation technique in the presence of poly vinyl pyrrolidone was successfully applied for the preparation of magnetite nanoparticles as core beads with narrow size distribution. The powders were characterized by $\mathrm{X}$-ray diffraction, particle size analysis, magnetic measurements, atomic force microscope and also infrared spectroscopy. FTIR and CHNS results indicated that the gallate anion was actually adsorbed onto the surface of the magnetite nanoparticles. The release of the anion from the surface of the nanocomposite was found to be controllable by the selection of the release media.
\end{abstract}

Keyword: Controlled release; Anti-carcinogenicagent; Gallate; Magnetite nanoparticles. 